\title{
Front Matter: Volume 10904
}

, "Front Matter: Volume 10904," Proc. SPIE 10904, Laser Resonators, Microresonators, and Beam Control XXI, 1090401 (22 May 2019); doi: $10.1117 / 12.2531394$

SPIE. Event: SPIE LASE, 2019, San Francisco, California, United States 


\title{
PROCEEDINGS OF SPIE
}

\section{Laser Resonators, Microresonators, and Beam Control XXI}

\author{
Alexis V. Kudryashov \\ Alan H. Paxton \\ Vladimir S. Ilchenko \\ Editors
}

4-7 February 2019

San Francisco, California, United States

Sponsored and Published by

SPIE 
The papers in this volume were part of the technical conference cited on the cover and title page. Papers were selected and subject to review by the editors and conference program committee. Some conference presentations may not be available for publication. Additional papers and presentation recordings may be available online in the SPIE Digital Library at SPIEDigitalLibrary.org.

The papers reflect the work and thoughts of the authors and are published herein as submitted. The publisher is not responsible for the validity of the information or for any outcomes resulting from reliance thereon.

Please use the following format to cite material from these proceedings:

Author(s), "Title of Paper," in Laser Resonators, Microresonators, and Beam Control XXI, edited by Alexis V. Kudryashov, Alan H. Paxton, Vladimir S. Ilchenko. Proceedings of SPIE Vol. 10904 (SPIE, Bellingham, WA, 2019) Seven-digit Article CID Number.

ISSN: 0277-786X

ISSN: 1996-756X (electronic)

ISBN: 9781510624504

ISBN: 9781510624511 (electronic)

Published by

SPIE

P.O. Box 10, Bellingham, Washington 98227-0010 USA

Telephone +1 3606763290 (Pacific Time) · Fax +1 3606471445

SPIE.org

Copyright @ 2019, Society of Photo-Optical Instrumentation Engineers.

Copying of material in this book for internal or personal use, or for the internal or personal use of specific clients, beyond the fair use provisions granted by the U.S. Copyright Law is authorized by SPIE subject to payment of copying fees. The Transactional Reporting Service base fee for this volume is $\$ 18.00$ per article (or portion thereof), which should be paid directly to the Copyright Clearance Center (CCC), 222 Rosewood Drive, Danvers, MA 01923. Payment may also be made electronically through CCC Online at copyright.com. Other copying for republication, resale, advertising or promotion, or any form of systematic or multiple reproduction of any material in this book is prohibited except with permission in writing from the publisher. The CCC fee code is $0277-$ $786 \times / 19 / \$ 18.00$.

Printed in the United States of America by Curran Associates, Inc., under license from SPIE.

Publication of record for individual papers is online in the SPIE Digital Library.

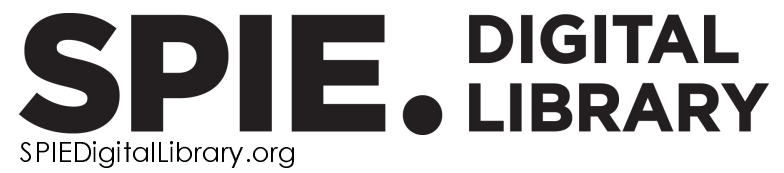

Paper Numbering: Proceedings of SPIE follow an e-First publication model. A unique citation identifier (CID) number is assigned to each article at the time of publication. Utilization of CIDs allows articles to be fully citable as soon as they are published online, and connects the same identifier to all online and print versions of the publication. SPIE uses a seven-digit CID article numbering system structured as follows:

- The first five digits correspond to the SPIE volume number.

- The last two digits indicate publication order within the volume using a Base 36 numbering system employing both numerals and letters. These two-number sets start with $00,01,02,03,04$, 05, 06, 07, 08, 09, OA, OB ... 0Z, followed by 10-1Z, 20-2Z, etc. The CID Number appears on each page of the manuscript. 


\title{
Contents
}

\author{
vii Authors \\ ix Conference Committee
}

MICRORESONATORS IN NOVEL DEVICES AND TOPOLOGIES I

1090402 Experimental observation of above billion quality factor in silicon crystalline optical whispering gallery mode resonators [10904-1]

1090403 Quasi-phase matching in integrated lithium-niobate whispering galleries (Invited Paper) [10904-2]

1090406 Enhancing Raman lasers with single molecule monolayers (Invited Paper) [10904-5]

MICRORESONATORS AND FREQUENCY COMBS I

10904 0C Advances in quantum optical frequency combs [10904-10]

10904 OD High repetition rate frequency comb up- and down-conversion in synchronously driven $\mathbf{x}^{(2)}$ microresonators [10904-1 1]

10904 OG Frequency comb generation in a quadratic nonlinear waveguide resonator [10904-14]

MICRORESONATORS AND SOLITONS I

$109040 \mathrm{~J} \quad$ Dual comb generation in a monochromatically driven crystalline microresonator [10904-17]

10904 OK Spectrum collapse, narrow lines, and soliton combs with multi-frequency laser diodes locked to optical microresonators (Invited Paper) [10904-18]

MICRORESONATORS AND SOLITONS II

10904 OM Kerr comb generation in a mode coupled system (Invited Paper) [10904-20]

$1090400 \quad$ Multiplexing soliton-combs in optical microresonators [10904-22]

10904 OP Dually-pumped Kerr microcombs for spectrally pure radio frequency signal generation and time-keeping [10904-23] 
10904 OS Observation of novel optical and microwave power dependent effects in silicon micro-ring modulator based frequency comb generators [10904-26]

$10904 \mathrm{OV} \quad$ Numerical studies on Kerr comb generation in $\mathrm{Si}_{3} \mathrm{~N}_{4}$ resonators with frequency dependent access coupler properties [10904-29]

MICRORESONATORS IN NOVEL DEVICES AND TOPOLOGIES II

$109040 Z$ Three-dimensional organic microlasers [10904-33]

QUANTUM OPTICS WITH MICRORESONATORS

1090413 Photon-mediated interactions between quantum emitters in a diamond nanocavity (Invited Paper) [10904-37]

MICRORESONATORS IN NOVEL DEVICES AND TOPOLOGIES III

1090416 Cavity-ringdown-spectroscopy-based study of high Q resonators in add-drop configuration [10904-40]

1090417 Passive and active whispering gallery mode microresonators in optical engineering (Invited Paper) [10904-41]

MICRORESONATORS IN NOVEL DEVICES AND TOPOLOGIES IV

10904 1A Characterization of the thermo-optic coefficient of silicon oxynitride using whispering gallery mode optical microcavities [10904-44]

$109041 \mathrm{C}$ Cross-polarization coupling of whispering-gallery modes due to the spin-orbit interaction of light [10904-46]

10904 1D Enhanced absorption sensing using non-adiabatic tapered fiber coupling to a whispering-gallery microresonator [10904-47]

iv 
BEAM SHAPING I

$109041 G \quad$ Beam shaping for ultrafast materials processing (Invited Paper) [10904-50]

$10904 \mathrm{1H} \quad$ High power laser mode conversion with volume phase elements recorded in PTR glass [10904-51]

$1090411 \quad$ Focusing laser beam through pinhole using bimorph deformable mirror [10904-52]

BEAM SHAPING II

10904 1K Stacked-actuators deformable mirror vs bimorph mirror for laser beam shaping [10904-54]

$109041 \mathrm{~L} \quad$ Design and analysis of binary fan-out gratings based on step-transition perturbation approach [10904-55]

$109041 \mathrm{M}$ Machine learning aided phase retrieval algorithm for beam splitting with an LCOS-SLM [10904-56]

BEAM COMBINING, MODE CONTROL, BEAM DIAGNOSTICS

$109041 \mathrm{~N} \quad$ Kramers-Kronig self-phasing effect in passive beam combining resonators (Invited Paper) [10904-57]

10904 IP Intracavity second harmonic generation for higher-order laser modes [10904-59]

$109041 \mathrm{R} \quad$ Novel method to mitigate ghost images in laser beam diagnostic and laser vision systems (Invited Paper) [10904-62]

\section{POSTER SESSION}

$109041 \mathrm{~T} \quad$ Numerical and experimental study of the dynamics of cross polarization coupling in a single whispering-gallery microresonator [10904-65]

$109041 \mathrm{~N} \quad$ Non-resonant operation of microcavity Brillouin lasers [10904-66]

$109041 \mathrm{~V} \quad$ Tapered hollow annular core fiber coupled whispering-gallery mode microsphere resonators [10904-67]

$10904 \mathrm{lW} \quad$ Sensing of multiple parameters with whispering gallery mode optical fiber micro-resonators [10904-68] 
$109041 \mathrm{Z}$ Conical refraction lasing in a Nd:YVO 4 laser with a conerefringent KGW element [10904-71]

Proc. of SPIE Vol. 10904 1090401-6

Downloaded From: https://www.spiedigitallibrary.org/conference-proceedings-of-spie on 25 Apr 2023 Terms of Use: https://www.spiedigitallibrary.org/terms-of-use 


\section{Authors}

Numbers in the index correspond to the last two digits of the seven-digit citation identifier (CID) article numbering system used in Proceedings of SPIE. The first five digits reflect the volume number. Base 36 numbering is employed for the last two digits and indicates the order of articles within the volume. Numbers start with 00, 01, 02, 03, 04, 05, 06, 07, 08, 09, 0A, OB...0Z, followed by 10-1Z, 20-2Z, etc.

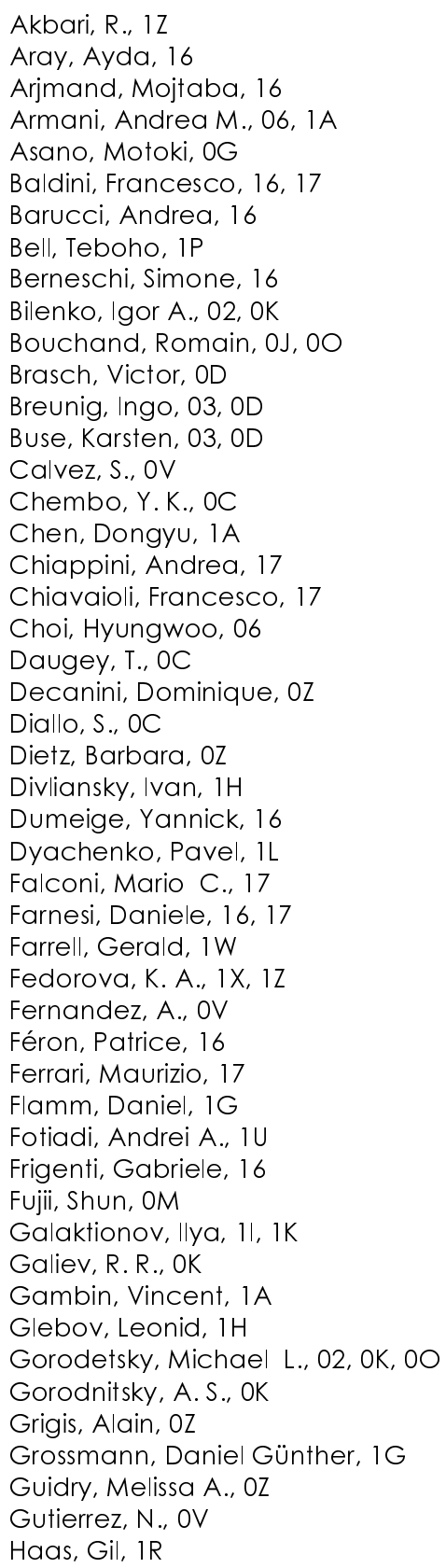

Hale, Evan, $1 \mathrm{H}$

Hellstern, Julian, 1G

Hermerschmidt, Andreas, $1 \mathrm{~L}$

Herr, Simon J., OD

Herr, Tobias, OD

Howlader, C., 1X, 12

Ikuta, Rikizo, OG

Imoto, Nobuyuki, OG

Jenne, Michael, $1 G$

Jeyaselvan, Vadivukkarasi, oS

Jia, Yuechen, OD

K.P., Nagarjun, OS

Kaiser, Myriam, $1 G$

Karpov, Maxim, 00

Kavungal, Vishnu, $1 \mathrm{~W}$

Ke, Limu, 1D, $1 T$

Kiedrowski, Thomas, 1M

Kim, Dong Cheon, 1L

Kippenberg, Tobias J., 0J, 00

Kishi, Tetsuo, 17

Kleiner, Jonas, $1 \mathrm{G}$

Kompan, Fedor, $1 \mathrm{H}$

Kondratiev, Nikita M., 02, OK

Koptyaev, S., OK

Korobko, Dmitry A., $1 \mathrm{U}$

Kovach, Andre, 1A

Kudryashov, Alexis, 11, $1 \mathrm{~K}$

Kumkar, Malte, $1 \mathrm{G}$

Kunkel, W. Minster, $1 \mathrm{~N}$

Lafargue, Clément, $0 z$

Laneve, Dario, 17

Lasagni, Andrés F., $1 \mathrm{M}$

Lebental, Mélanie, $0 Z$

Lecomte, Steve, OD

Leger, James R., $1 \mathrm{~N}$

Lihachev, Grigory, 00

Llopis, O., OV

Lobanov, Valery E., 02, OK

Lucas, Erwan, $0 \mathrm{~J}, 00$

Major, A., 1X, 12

Mallik, Arun Kumar, IW

Matsko, Andrey B., OP

Merolla, J.-M., OC

Mikhaylov, Dmitriy, 1M

Mikut, Ralf, $1 \mathrm{M}$

Nadimi, M., 1X

Nazabal, Virginie, 17

Ngcobo, Sandile, $1 \mathrm{P}$

Nikitin, Alexander, 11, 1K 
Nunzi Conti, Gualtiero, 16, 17

Obrzud, Ewelina, OD

Palma, Giuseppe, 17

Pavlov, Nikolay G., 0K, 00

Pelli, Stefano, 16

Piveteau, A., 0C

Poust, Sumiko, $1 \mathrm{~A}$

Prudenzano, Francesco, 17

Rafailov, E. U., 1X, 1 Z

Raj, Piyush, OS

Raja, Arslan S., 00

Rajagopal, Sreekul Raj, 1D, IT

Righini, Giancarlo C., 16

Rosenberger, A. T., 1C, 1D, $1 T$

Rukosuev, Alexey, 11, 1K

Samarkin, Vadim, 11, 1K

Scaggs, Michael, 1R

Scarnera, Cosimo, 17

Scharf, Toralf, 1L

Selvaraja, Shankar Kumar, OS

Semenova, Yuliya, IW

Sheldakova, Julia, 11, 1K

Shen, Xiaoqin, 06

Shitikov, Artem E., 02

Sokolovskii, G. S., 1X, 1 Z

Sokolovskii, S. G., $1 \mathrm{U}$

Soltani, Soheil, $1 \mathrm{~A}$

Song, Yalei, $\mathrm{OZ}$

Soria, Silvia, 16, 17

Sukachev, Denis D., 13

Supradeepa, V. R., OS

Suzuki, Ryo, OM

Szabados, Jan, OD

Taheri, Hossein, OP

Tanabe, Takasumi, OM

Tani, Ryoya, 0G

Tillkorn, Christoph, 1G

Toporovsky, Vladimir, 1K

Voloshin, Andrey S., 02, OK

Wang, Jiawei, $1 \mathrm{~V}$

Wang, Tingyun, $1 \mathrm{~V}$

Waritanant, T., $1 \mathrm{X}$

Weng, Wenle, OJ

Xiao, Hai, $1 \mathrm{~V}$

Yamamoto, Takashi, OG

Yang, Yong, $1 \mathrm{~V}$

Zhang, Xiaobei, $1 \mathrm{~V}$

Zhou, Baifan, IM

Zimmermann, Felix, 1G

Zolotovskii, Igor O., $1 \mathrm{U}$ 


\section{Conference Committee}

Symposium Chairs

Beat Nevenschwander, Berner Fachhochschule Technik und Informatik (Switzerland)

Xianfan Xu, Purdue University (United States)

Symposium Co-chairs

Koji Sugioka, RIKEN Center for Advanced Photonics (Japan)

Reinhart Poprawe, Fraunhofer-Institut für Lasertechnik (Germany)

Program Track Chairs

Vladimir S. Ilchenko, GM Cruise LLC (United States)

Paul O. Leisher, Lawrence Livermore National Laboratory (United States)

Conference Chairs

Alexis V. Kudryashov, Institute of Geosphere Dynamics

(Russian Federation)

Alan H. Paxton, Air Force Research Laboratory (United States)

Vladimir S. Ilchenko, GM Cruise LLC (United States)

Conference Co-chair

Andrea M. Armani, The University of Southern California (United States)

Conference Program Committee

Lutz Aschke, TRUMPF Lasertechnik GmbH (Germany)

Gaurav Bahl, University of Illinois (United States)

Paul E. Barclay, University of Calgary (Canada)

Hui Cao, Yale University (United States)

Yanne K. Chembo, University of Maryland, College Park (United States)

Jean-Claude M. Diels, The University of New Mexico (United States)

Hans Joachim Eichler, Technische Universität Berlin (Germany)

Andrew Forbes, University of the Witwatersrand, Johannesburg (South Africa)

Pierre Galarneau, INO (Canada)

Michael L. Gorodetsky, Russian Quantum Center (Russian Federation)

Thomas Graf, Universität Stuttgart (Germany)

Qing Gu, The University of Texas at Dallas (United States)

Stefan Hambücker, INGENERIC GmbH (Germany) 
Tobias J. Kippenberg, Ecole Polytechnique Fédérale de Lausanne (Switzerland)

James R. Leger, University of Minnesota, Twin Cities (United States)

Andrey B. Matsko, OEwaves, Inc. (United States)

Gualtiero Nunzi Conti, Istituto di Fisica Applicata "Nello Carrara" (Italy)

Andrew W. Poon, Hong Kong University of Science and Technology (Hong Kong, China)

Michael J. Scaggs, Haas Laser Technologies, Inc. (United States)

Julia V. Sheldakova, Russian Academy of Sciences (Russian Federation)

Haiyin Sun, Chemlmage Corporation (United States)

Yun-Feng Xiao, Peking University (China)

Lei Xu, Fudan University (China)

Jonathan M. Ward, Okinawa Institute of Science and Technology

Graduate University (Japan)

Lan Yang, Washington University in St. Louis (United States)

Session Chairs

1 Microresonators in Novel Devices and Topologies I

Vladimir S. Ilchenko, GM Cruise LLC (United States)

2 Microresonators in Sensors

Andrea M. Armani, The University of Southern California (United States)

3 Microresonators and Frequency Combs I

Andrey B. Matsko, OEwaves, Inc. (United States)

4 Microresonators and Solitons I

Tobias J. Kippenberg, Ecole Polytechnique Fédérale de Lausanne (Switzerland)

5 Microresonators and Solitons II

Yanne K. Chembo, University of Maryland, College Park (United States)

6 Microresonators and Frequency Combs II

Jonathan M. Ward, Okinawa Institute of Science and Technology Graduate University (Japan)

7 Microresonators in Novel Devices and Topologies II

Jonathan M. Ward, Okinawa Institute of Science and Technology Graduate University (Japan)

8 Quantum Optics with Microresonators

Vladimir S. Ilchenko, GM Cruise LLC (United States) 
9 Microresonators in Novel Devices and Topologies III

Alan H. Paxton, Air Force Research Laboratory (United States)

10 Microresonators in Novel Devices and Topologies IV

Vladimir S. Ilchenko, GM Cruise LLC (United States)

11 Beam Shaping I

Stefan Hambücker, INGENERIC GmbH (Germany)

12 Beam Shaping II

James R. Leger, University of Minnesota, Twin Cities (United States)

13 Beam Combining, Mode Control, Beam Diagnostics

Alan H. Paxton, Air Force Research Laboratory (United States) 
Proc. of SPIE Vol. 10904 1090401-12 Downloaded From: https://www.spiedigitallibrary.org/conference-proceedings-of-spie on 25 Apr 2023
Terms of Use: https://www.spiedigitallibrary.org/terms-of-use 